\title{
Design and Analysis of Spatial Skyline Queries Indexing
}

\author{
Swathi Sowmya Bavirthi, Supreethi. K. P
}

\begin{abstract}
Dwelling in the information age permits nearly everybody needs to recover countless information and choices to gather from to fulfill their necessities. In distinctive cases, the quantity of information accessible and the speed of change may cover the ideal and required explanation. Spatial-textual queries provide the most acclaimed nearest points concerning a conveyed site and a keyword set. Current practice regularly thought on the most capable technique to expertly get the top-k resultset reestablished a spatial-scholarly query. A capable Spatial Range Skyline Query (SRSQ) algorithm is proposed which initially performsa spatial keyword query (SKQ) that relies upon an IRtree that documents the information. Skyline centers picked are not simply established on their partitions to a lot of inquiries and more subject to their significance to a social occasion of query keywords. Additionally, besides proposed range skyline (RS) methods based on R-tree multi-dimensional space including secondary- memory pruning tools for operating field skyline queries is accomplished. The advanced scheme is dynamic and I/O optimum. Ultimately, methodology presents a modern assessment that demonstrates the proficiency.
\end{abstract}

Keywords: R-Tree, road networks, Nearest Neighbour, Indexing, Textual relevance, maximal $k$ spatial patternqueries.

\section{INTRODUCTION}

With the fast improvement of versatile handset gadgets (example: Cell phones and tablet PCs), remote correspondence, and situating advances within the earlier ten years. Location-based services (LBSs) have prospered. Customers utilizing area aware cell phones can query LBSs concerning points of interest (POIs) anyplace and at all focuses. Among the few types of locale-based deals, one tremendous class is a region-based horizon. Theseinquiries consider together the spatial and non-spatial components from these POIs [6], [15]. An agent model is finding adjacent eateries with great sustenance, where the questioning client is part of something spatial trademark and the constancy of the sustenance is a nonspatial quality. The inquiry re-establishes a lot of bistros that are closer to the questioning client or perhaps have the best sustenance over those not returned.

The spatial objects can have an expansive once-over of non-spatial characteristics, for example, sustenance quality, association, tidiness, condition, and cost-only a little subset of these credits is basic to a specific client in the alone query. Closest neighbor (NN) search [1], [3], turn around $\mathrm{NN}$ search is delegate ones[2]. Over that spatial request, non-spatial highlights of articles might be seen as together as extra yet free query designs.

Revised Manuscript Received on March 18, 2020.

Swathi Sowmya Bavirthi, Asst.Professor, IT Department, Chaitanya Bharathi Institute of Technology(A), Hyderabad, Telangana State, India

Dr. Supreethi.K.P, Professor, CSE Department, JNTUH College of Engineering, Hyderabad, Telangana State, India.
For the event, a query"finding the closest motel with a cost underneath $\$ 250 "$ utilizes inn cost, to distill economical lodgings over the NN search. Another query "obtaining the scarce precious inn inside a mile" obtains the most limited upscale inn inside a scale query sequence.Spatial information denotes contrarily described geographic data. Spatial numbers are usually put continuously as controls also, topology and data, to be able to be mapped. A spatial geodatabase is a database that occurs improved to assemble and query statement that holds an eye on things described, that leads to element outlined in a geometric position to the highest intensity spatial databases authorize discoursing to an essential geometric entity.Area-based services are the services offered to clients dependent on their areas. Areabased services have numerous different applications like finding the closest stores in a specific region, area-based promotion, breaking down untamed life and traffic developments, area-based gaming, and so forth. The movements in the database and adaptable advancement and rapidly extending conspicuousness of area-based services bring about gigantic measures of information being gathered in databases. Area-based organizations have pulled in basic thought from the mechanical and research network. Help hardware to Cloud service providers (CSPs) for embodies, Amazon EC2 and Google Application Engine [29]. Altogether more unequivocally, an information owner doles out its information to a CSP, which thusly gives inquiry connections to customers considering a genuine worry for the information owner. While such a redistributing model is useful to the extent cost, execution, and versatility in resource the board, it conveys an unbelievable test to question trustworthiness affirmation [30], [31].

A structure for ensuring zone security of a customer have described three sorts of Nearest Neighbor requests, specifically, open request over private information, mystery question over open information and masterminded inquiry over mystery information.

Another example for spatial skyline is when restaurant store owner trying to find customers who have the vegetarian restaurant in its $\mathrm{k}$ nearest neighbours for sending them discount coupons. An example of Private Query over Public Data is a user searching for a good location to stay such that the customer is in the influence of at least $\mathrm{k}$ facilities like School, Hospitals, etc. Examples of Private queries over Private Data include peer to peer applications like file sharing and match-fixing applications were a match-fixer wants to avoid an area where there are other match-fixers around him.

Actually, a large number of the present applications would think about both item closeness furthermore, their nonspatial qualities as item choice and arranging criteria, at the same time. Consequently, numerous ongoing exploration works, for example, [4], [5], [6], [7], [8], [9] have investigated the thought of location predominance in 
Location-subordinate data get to.Many similar problems and operators related to horizon questions have been observed in the examination. For instance, the Top-K query [10] recovers the best $\mathrm{K}$ receivers that determination of confinement particular inclination work. The distinction from the horizon query is that the yield changes as indicated by a client determined input function and the recovered focuses are not an important piece of the horizon. The knearest neighbor (k-NN) query [11], in another example, requires the existence of an inquiry point $\mathrm{p}$ and yields the $\mathrm{k}$ protests closest top, in expanding request of their separation. In this case, the difference from the skyline query is that the $\mathrm{k}-\mathrm{NN}$ query recovers answers as indicated by the nearness of a given point and not based on domination to other points. Finally, convex hull $[12,13]$ contains the points that are enclosed by the polygon that is defined from the minimum and maximum skyline (i.e. based on the evaluation criteria the minimizing and maximizing values are decided) of the given set of points. The main difference from a skyline is that it defines an area of interest instead of a line with individual interesting point.

The aim is to propose an approach for evaluating spatialtextual skyline queries using indexing. The objectives are:

- The introduce top-k spatial keyword queries approaching street arrangements.

- Fordescribingthe novel catchphrase adjusted spatial watchword top-k query shrinkage to a query filtration problem.

- Design algorithm reliant on a document structure, this picks off the most catchphrase set among a lot of contenders utilizing a memory pruning methodology.

Finally, should be play out a test assessment that shows the proficiency of the methodology.

\section{RELATED WORKS}

Skyline inquiries were considered widely in multidimensional spaces in metric spaces, in one of kind spaces, in spilling circumstances, and in time-course of action data. A few algorithms[14] proposed for skyline query taking care of, for instance, window-based, dynamic, appropriated, geometric-based, record-based, isolate andendure, and dynamic programming calculations. Additionally, a few varieties proposed to take care of using explicit issues like the k-overwhelming skyline, top-k ruling inquiries, and spatial skyline queries, moreover distinct.[15] handled visit developments of the objects being questioned, which additionally proposed steady forms of I-SKY including N-SKY, which declined to recompile the query statement and results without any preparation. The strategies for noticing subspace skyline query on high dimensional information to such an extent [16], that both dematerializations hoarding and question time can be facilitated. This is the propose astute considerations of maximal generally teaching space, the maximal worth space between sets of skyline query in the full space and use these thoughts as the foundation for seeing subspace skyline demand high dimensional information.

Index-based approaches [17] are specific with an increased R-tree and predominance chart. Enlarged R-tree enlargesthe tree by carrying no spatial aspects to archive required focuses to connect with predominance checks during onceover traversal. Strength is graph-related with a recomputed no predominance scope wherein query focuses/foci ought to have the comparing object, not location overwhelmed by some other.NN (nearest neighbors) was an algorithm that applies the gap and-overcome system on datasets document by R-trees. NN did any alluring highlights.

BBS called the branch and bound skyline was apowerful algorithmin a like manner subject to nearest neighbor search that was IO perfect [18]. BBS was implemented and productively connected to an assortment of elective skyline queries.

An efficient algorithm for processing spatial-skyline STDbased skylines described in [19] which models sexually transmitted disease and spatial separation with printed significance in a determined dimensional space.

A turnaround skyline query dealing with the plan associated with a general skyline and considers estimation spaces. That strategy [20]prepared invert skyline questions in the measurement spaces utilizing the current spatial ordering plan and considers both monochromatic and bichromatic conditions.

MB-tree[21], another list structure for spatial information, its striking information assigning framework was the establishment ofthe MBSQ algorithm. Segment data space as indicated by the $\mathrm{x}$ organize and $\mathrm{y}$ arrange on the other hand until the quantity of data in each MBR existed not as much as $\mathrm{M}$.

The layer-based methodology[22] pre-forms tuples into decided layers. The methodology constructs a double objectives layer that involves coarse-level and fine-level layers. In particular, they assemble coarse-level layers utilizing skylines and portion each coarse-level layer into fine-levelsub layersutilizing curved horizons. Then the resulting optimization intentions were addressed as: 1) record-making; 2) disk-based storage policy; 3) study of the virtual layer, and 4) index preservation for tuple modified.

R-kNN (Reverse k-Nearest Neighbour)query discovers applications in decision supportive networks where the task is to open a new facility like a restaurant in an area such that it will be least influenced by its competitors and attract good business. Another application is profile-based marketing, where a company maintains profiles of its customers and wants to start another administration with the end goal that the administration is affected by the greatest number of clients. The R-kNN query in the decision support system is a precedent of a monochromatic query as the database gadgets and the query are of a similar kind. The use ofR-kNN in profile-based marketing is an example of a dichromatic query as the database objects are customers and the query is service to be started by the company.

The skyline query over Least Jumping Square shapes (MBRs) gets a lot of MBRs and returns the MBRs that were not dominated by different MBRs. In the predominance test for MBRs [23], the nitty-gritty characteristic estimations of objects in the MBRs were not gotten too.

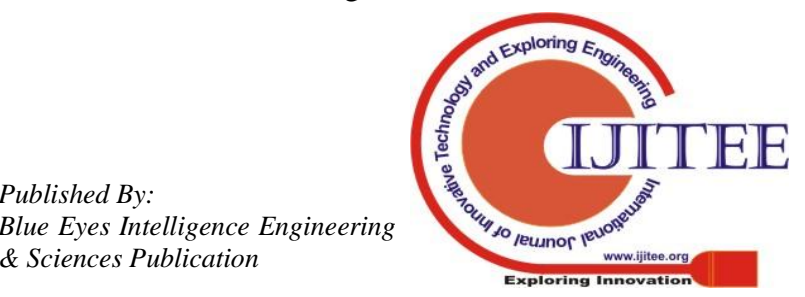


Also, the reliant gathering of MBRs lessens the quest space for strength tests. Objects in an MBR were just contrasted and the ones in the relating ward gatherings of the MBR instead of with the whole dataset. Their answers apply the two ideas to the R-tree so as to utilize its progressive structure in which each hub is a characteristic reflection of an MBR. In particular, given the R-tree record of an information dataset, they originally wiped out unfit objects by using the skyline query over MBRs.

An all-inclusive profundity first search ordering strategy (eDFS for short)[24], introduced to exhibit the presentation and utility of our ordering technique, for getting to client inclination profiles similar todirected acyclic graphs(DAGs), the structure of the e-DFS encoding that satisfactorily encodes a customer inclination profile into a lowdimensional point which will be listed by a R-treein the long run.

The strongly prune non-skyline focuses on an intriguing space. Two improvedmethodologies [25] contributed to reduce the number of division estimations and power tests proposed over spatial-skyline query issue, where the majority of the centers were situated in few-dimensional Euclidean spaces. It is moreover ardently related to the nearest neighbor (NN) query.

The implementationpruning procedure was introduced to develop the query proficiency given user region [26]; a limit theta, and numerous keywords, skyline and p-skyline objects situated at various bearings have come back to the client. Distinguishing the outcome relies upon three viewpoints: spatial distance, printed similitude, and course.

The displayed versatile algorithm [27] discover adjusted parcels utilizing both the spatial and sequence data inserted tirelessly in the treesearch word in both Euclidean time andstreet systems. The algorithm is focusing on queryspecific spatial conjectured spatial approximate string (SAS) query. In Euclidean time, space, the MHR-tree produces min-wise illustrations into an R-tree. The min-wise reproduction for a record center point ukeeps a short portrayal of the relationship of q-grams from strings under the subtree of $\mathrm{u}$. Pruning comfort of such checks subject to the set similarity interfacing the query string and the qgrams from the subtreesoflist hubs is discussed.

\section{METHODOLOGY}

In this segment, algorithm forSpatial Range Skyline Query (SRSQ) is proposed, which uses native algorithm for counting Spatial Keyword Query (SKQ) by applying IR tree for index data and Range Skyline (RS) methods execute using R- tree multi-dimensional secondary memory pruning tool and operates on skyline queries.

SKQ is a sequential scan based algorithm (SS), dependent on the user query [32]. For a query, SS algorithm startsfrom the recommendedassessments ofevery single inferred measurement for each item. At that point, every objectis arranged in the climbing request of the aggregate of the estimations of their inferred measurements. Algorithm SS examines the arranged rundown also, checks whether everythingis in the skyline by confining it and the starting late discovered skyline objects. The wickedness of SS lies in figuring, movement and planning the estimations of each picked estimation for each article. For productivity, currently proposing an essential and propelled algorithm for SKQ.
To recognize areas for objects, in space of $\mathrm{S}$, and streamline the test for a thing to a fundamental thought search in specific regions of different gadgets. Specifically, for whatever object $\mathrm{d} € \mathrm{D}$ moreover the query areadk $€ S$, let $C^{d k}$ be the circle collected at $d k$ including radius $\operatorname{st}(q k ; d)$. Grant $\mathrm{W}$ signify the MBR of the entire dataset D. Formerly:

The ambiguity region of a gadget $\mathrm{d}$ is $\mathrm{Ru}(d)=$ $W_{q k \in Q} C^{d_{d k}}$. The dominance section of an object $\mathrm{d}$ stands $R d(d)=W-R u(d), d d^{2 o m} d^{\prime}$

Given a query $S$, the objects collected by the dominance area $\operatorname{Rd}(\mathrm{d})$ of $\mathrm{d}$ are commanded by d, i.e., $\forall d^{\prime} \in R d(d)=$ $W-R u(d)$

Equation 1: For any $d^{\prime} \in R d(d)$, they have $\forall q k \in$ $S, d\left(d k, d^{\prime}\right)>s t(d k, d) \quad$ Since $\quad w\left(S . \varphi, d^{\prime} . \varphi\right) \in$ $\left.[0,1], \operatorname{st}\left(d k, d^{\prime}\right)=d\left(d k, p^{\prime}\right) \mid w\left(S . \varphi, d^{\prime} . \varphi\right) \geq \operatorname{st}(d k, d)\right]$ Therefore, $\forall d^{\prime} \in R d(d)=W-R u(d) œ$. On the other hand, for any $d^{\prime} \in R d(d)$, there cannot confirm whether $d$ dominates $d^{\prime} œ$.

In Equation 1 canister be applied to clip a non-skyline object $\mathrm{d}$ constrained to the point area of a desolate conditiond. and the current Condition 2 such prunes non-skyline purposes appropriating the area of a number of objects.

Given a query $S$, think about a lot of objects $Q=\{d 1 ; d 2 ;$ : $::\}$.the uncertainty region of $\mathrm{A}$ is $R u(Q)=d i>$ $S^{\wedge} R u(d i)$.the region of $\mathrm{Q}$ is $R d(Q)=W-R u(Q)$. The objects inside $R d(Q)$ are not components of the skyline.

Equation 2: Suppose $\exists d \otimes R d(Q)$, such that not commanded by either object in Q. That indicates $d i \in$ $Q . d^{\prime} \in R u(d i)=d^{\prime} \in \cap p i \in Q R u(d i) C \cap d i \in$

$Q R u(d i)=R u(Q)$ this repudiates the accepting that $d^{\prime} \in$ $R d(Q)=W-R u(Q)$.

\section{A.Precursory: IR-Tree}

Themeasurement can be identified with any uncommon leveled spatial printed record, which encourages productive pursuit dependent on spatial literary significance to a reference object $q$. The IR-tree [35] is the cutting edge record in this class. Figure 1 watches out for the focal structure of a fantastic IR-tree. In our remarkable condition, the things from $\mathrm{E}$ are accumulated into the leaf central purposes of the snappy diagram; each leaf focus point in like manner has a pointer to a turned around record, which documents the substance depictions of the significant number of objects in it. A reworked record has two standard parts: (I) a jargon of every single unmistakable term showing up in the depictions of an object and (ii), this is the posting list for each term L, i.e., improvement of sets $(U ; t)$, where $U$ is the identifier of the thing in the leaf focus whose system contains $\mathrm{L}$ and $\mathrm{t}$ is the massiveness of $\mathrm{L}$ in the diagram. All non-leaf focus point contains the MBRs of its young people's focuses and in addition a pointer to a disturbing record mentioning the pseudo-depictions of its kid's hubs. Given a query d, involving a spatial area and a literary depiction $\mathrm{d}$, the MBR of an IRtree section can be utilized to pick a lower bound of the Euclidean parcel among $\mathrm{d}$ and any article recorded under the area. The pseudo-depiction of the bit can be utilized to figure an upper bound on the printed importance among q: and any things recorded underneath the section. 


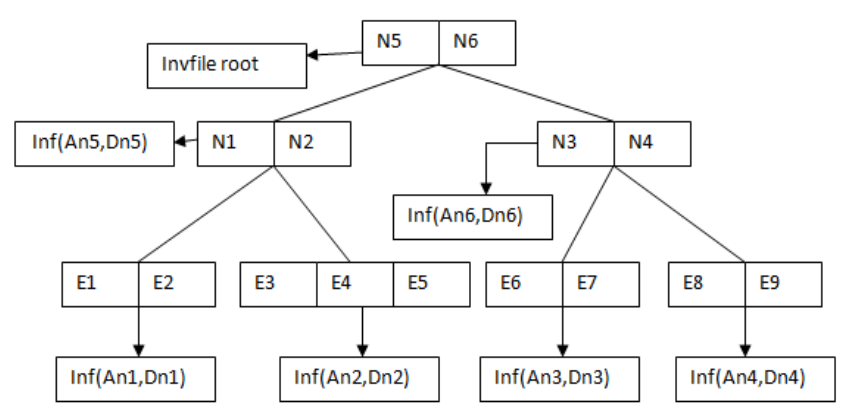

Figure: 1. Inverted files in IR-Tree

\section{B. Spatial Range Skyline Query for SKQ}

To currently propose a propelled the algorithm that utilizes an in-memory report construction, the skyRtree, and furthermore three pruning practices, with the intention that the I/O and CPU cost during the analysis of up-and-comer skyline markets is reduced.

\section{skyRtree}

The skyRtree is an in-memory R-tree that files each unit of the observed skyline objects in the reservation of SQS determined measurements. At the point when another skyline item is recognized, it is embedded into the skyRtree. Algorithm 1 SKQ (Query N, Dataset D, Index IRTree)

- $\quad$ Skyline particle $\mathrm{Q}=\emptyset ; \mathrm{A}=\mathrm{W}$

- $\quad$ Min Heap MH = $\varnothing$;

- Compute the root of IRTree to MH

- while MH is not blank do

- $\quad$ sub tree establish $\mathrm{c}=\operatorname{deHeap}(\mathrm{MH})$

- $\quad$ if c.MBR $\cap \mathrm{A} \neq \varnothing$; then, from eq 1 and 2

- $\quad$ check if e is a gadget before

- $\quad$ if Given a query $S \neq \varnothing$, then

- Compute c to Q

- $\quad \operatorname{record} \mathrm{A}=\mathrm{A} \cap \mathrm{Ru}(\mathrm{e})$

- else

- $\quad$ isSkyline $=$ True

- for each $\mathrm{d} \in \mathrm{Q}$ do

- ifdominance section of an object d domQ $\mathrm{c}$ then

- $\quad$ is Skyline $=$ False

- break

- if isSkyline then

- $\quad$ Add c to Q

- $\quad \mathrm{A}=\mathrm{A} \cap \mathrm{Ru}(\mathrm{e})$

- $\quad$ else note: $\mathrm{c}$ is a non-leaf entry

- $\mathrm{R}=$ readNode(e)

- for each entry cœ in $\mathrm{R}$ do

- $\quad$ if c.MBR $\cap \mathrm{A} \neq \varnothing$; then, then eq 1 and 2

- $A D D\left(e^{\prime}, \sum q i \in S^{s t\left(q i, e^{\prime}\right)}\right)$ to $M H$

- return Q

The MBRs of the skyRtreecenter points fill up in as rundowns for the skyline articles created persistently in the subtrees spoken to by them. Given query $N$, Let $Q=\{d 1$; $\mathrm{d} 2 ;:::\}$ be a gathering of effectively found skyline particle created by a skyRtree common feature. Let ${ }^{\wedge}$ be the MBR of $\mathrm{Q}$ in the $|\mathrm{Q}|$ - dimensional over time.Give pu a chance to be the highest corner of $\wedge$, characterized by the most extreme qualities in all $|\mathrm{Q}|$ estimations of the skyline objects. Formally, the kth estimation of $\mathrm{pu}$ is proportionate tomax $\forall \mathrm{di}>S$ st $(d k ; d i)$.

Statement 1 - If object $d$ is dominated by du, disn't a skyline object. Verification: Point du represents the most pessimistic scenario of the skyline objects inside MBR. On the off chance that an item is dominated by du, it necessity be ruled by every the skyline particle inside the MBR. The pruning intensity of an MBR at a lower level, using Clarification 1, is more grounded than that of an MBR at a higher level. The proposal connects with us to isolate a thing $\mathrm{p}$ and the uppermost corner of an MBR to prune d.Give dl a chance to be the lower-most corner of MBR jumping Q. Officially, the kth estimation of du is equivalent tomin $\forall d i \in \mathrm{S} s t$ (sk; di) Proposition 2 uses du of the root MBR of the skyRtree to affirm whether an object is in the skyline.

Statement 2- Let dur be the lower-most circle of the root MBR. In the event that dur can't dominate object $d, d$ is a skyline object. Verification: Point DLR represents the best instance of all the found skyline objects. On the off chance that an item can't be dominated by DLR, none of the uncovered skyline objects can overpower it. Starting now and into the foreseeable future, it is a skyline object. Proposal 3 uses dl of the MBRs of non-leaf sky tree areas toabstain from looking at the sky tree focuses recorded under them with applicant skyline objects.

Statement 3- Give dl a chance to be the bottommost enclose of the MBR bouncing $\mathrm{Q}$. In the event that dl does not rule a contender skyline object $d$, at that point $d$ isn't commanded by several skyline objects in $\mathrm{Q}$ and necessities not to be separated and them. The affirmation is unimportant and blocked. The normal objective of Clarification 1,2, and 3 is to lessen the correlations test needed for any challenger skyline particle $\mathrm{d}$. Revelation 1 can propositionally be correlated to pruning several particles, whose MBR in the preferred location is led by du.and by the keep on introducing the decided segments of the non-leaf regions in the IR-tree. Utilizing that addition, an IR-subtree receiving a lot of particles might be pruned appropriatingStatement 1 .

Each time another skyline article is found, it is embedded into the skyRtree (stage 12). Capacity R-TREEPRUNES Algorithm 2 is utilized to prove whether a solitary a piece or a subtree repository is pruned varieties leave 9 and 15 . It performs the skyRtree and the pruning procedures to choose whether the information parameter d (a solitary item or many objects in a subtree) canister be defeated or not. The skyRtreereportevery one of the skyline objects known up until now. Capacity R-TREEPRUNES returns genuine if the information $\mathrm{d}$ is dominated. The skyRtree is navigated in an expansiveness first way. First and foremost, the info parameter $\mathrm{d}$ is contrasted and the corners Dr and Dur of the root MBR. In the event that Dr does not dominate $d$, at that point none of the skyline objects in the skyRtree rump check $\mathrm{d}$ and false is delivered byStatement2. On the off chance that Dur dominates d, at that point every the skyline objects in the skyRtree can overpower $d$ and veritable is returned by explanation 1. On the off chance that not of the preceding cases operates a Queue is designed and the base of the skyRtree. R-TREEPRUNES goes over the going with framework until the Line is unfilled. The main segment $R$ in Line is removed for assessment. On the off chance that $R$ is a non-leaf hub, for every section e in R, on the off chance that Duc of e dominates $d$, at that point $d$ must be commanded by the skyline centers in the subtree appended at $\mathrm{c}$ as per articulation 1 . In this way, the capacity returns genuine. Something else, if Drcof dominates d, all of the passages in $\mathrm{R}$ are skyline particles and there examine whether $\mathrm{d}$ is identified by those skyline particles. For this situation, the division period results condition, the breaking point returnsinvestigation. 
On the off chance that Drc does not dominate $d$, the sub tree established at $\mathrm{c}$ can be disposed of with no further handling as per Recommendation 3. On the off chance that $\mathrm{R}$ is a leaf center point; the majority of the segments in $\mathrm{R}$ are skyline objects furthermore to verify whether $d$ is inspired by these skyline objects. For this situation, the numerous advanced interestsgenuine. At the point when the Queue ends up the void, this implies d isn't overwhelmed by any skyline item and dishonest is delivered.

Algorithm 2 R-TREEPRUNES (d)

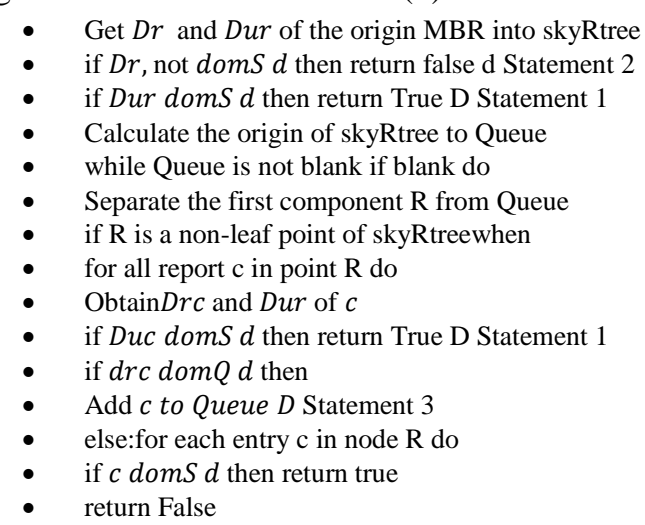

\section{Two-Dimensional Query Spell}

The R-tree [36] will fill in as the spine spatial store informationto prepare the range skyline query. The proposed algorithm uses a spatial skyline query in a range skyline technique to prune the fascinating space, as does the algorithm for the point-based unique skyline query[37]. The pseudo-code regarding this algorithm for maintaining the range skyline query i.r.t. a 2-d indicating iso-planned conventional appearance $\Delta$ is described in Algorithm 2, which is an alteration of Algorithm 1 for 2-d query intersection patterns $\Omega$. The algorithm applies two essential principles $\mathrm{H}$ and $\mathrm{H}^{\prime}$ with sets $\langle\mathrm{c}, \mathrm{Dc}\rangle$, where e is an R-tree region and $\mathrm{Dc}$ is the sub-area of $\Delta$, i.r.t. which e has not yet been allowed to be truly constrained by some other data point. The sections in $\mathrm{G}^{\prime}$ are performed in the upcoming presentations of the base detachment of Dc to qs. The areas in $G$ are constituted in the rising advising of the base departure of $c$ to the query time dcs $\in$ Dc, established at the base improbable ways from qs.

Algorithm 3: The Range Skyline RS method of the 2-d query (D)

Input: $\mathrm{A}$ dataset $\mathrm{D}$ recorded utilizing an $\mathrm{R}$-tree $\mathrm{N}$ also the query $\Delta$ [qs, qe]

- $R S=\emptyset, G=G^{\prime}=\varnothing$

- Insert into $G$ agroup of the form $\langle c, \Delta\rangle$ for each entry $c$ in origin of $\mathrm{N}$

- $\quad$ Repeat

- $\quad$ While $G=\varnothing$ Do

- Remove top entity pair $\langle c, D c\rangle$ of $\mathrm{G}$

- If $\exists D c c \in D e: \nexists<q, D q\rangle \in R S$ For Which $D c \in$ $D q$ and $q<D c c$ Then

- If [The point access of Dcc with the smallest length to $d s$ ] [the time of $d c s$ of Dc with the smallest length of $d s]$ Then Enter <> into G.

- Else

- If $\mathrm{c}$ does an MBR when

- $\quad$ For each drive child cc of $c$ do

- If $\exists D c c \in D e: \nexists<q, D q\rangle \in R S$ For Which $D c \in$ $D q$ and $q<D c c$ then
- If [The purpose of including some smallest measure to ] [the design of $d c s$ of Dc with the base separation of $d s]$ Then Insert $\langle c c, d c c c\rangle$ into $\mathrm{G}$

- Else

- $\quad$ For every pair $\langle d, D q\rangle$ in RS for which $D c c \cap D q \neq \emptyset$ Do

- If there is sub rectangle $\Delta q q \in(D q \cap D c c): c<\Delta q q P$ then

- $\quad$ Update $<d, D q-\Delta q q$ - $>$ into RS

- Insert $\langle c, D c c>$ into RS

- Move into $\mathrm{G}$ the pair of $\langle c, D c\rangle \in G^{\prime}$ having the region Dc with the minimum distance to $\mathrm{ds}$;

- Until the $G^{\prime} \neq \varnothing$;

- $\quad$ Return RS

The first execution of the circle in quite a while looks at regardless of regardless of whether there are data cores that are changing skyline turns about i.e. asub region of $\Delta$ which associates the base left-most end-point qs of $\Delta$. Absolutely when the load $\mathrm{G}$ discharges just because at that point the assistant pile $\mathrm{G}^{\prime}$ will includeevery one of the R-tree sections which are competently managed by data focuses in RS $\Delta$ i.e.qs at any rate they most likely won't be powerfully commanded i.e. each case in $\Delta$. In methodology 19 , the algorithm determines and advises from $G^{\prime}$ to $G$ every one of the sets $\langle\square,>\in \square$ ', the locale Dc of which is situated at the base good ways from qs. On the off chance that various districts Dc exist at a similar least good ways from qs yet with an alternate query point $\square \square \square \in \square \square$, which is situated at the base good ways from ds, at that point just the group of passages of ' $\mathrm{H}^{\prime}$ having similar DCS are encouraged into $\mathrm{H}$ and the rest of the territories of $\mathrm{H}^{\prime}$ are taken care of in a scheduled performance of the buoy in Lines 3-20. While it is located in arrangement 20 that stacks $\mathrm{H}$ and $\mathrm{H}^{\prime}$ are empty, the algorithm stops and the RS $\Delta$; firm contains records of the composition $\langle q, D q\rangle$, in which $p$ is novel skyline speculation after $\mathrm{Q}$ i.e. the sub-zone $\mathrm{Qp} \square \Delta$; in that boundary, $\mathrm{p}$ is a range skyline definition following $\mathrm{Q}$ i.r.t. $\Delta$.

\section{RESULTS AND DISCUSSION}

Anadvanced SRSQ algorithm is assessed and separates at available required data. For assessing the ability of SKQ and RS, different parameter environments, viz. leaving the balance of query fields, the dimension of query signals, the spatial design of query terms, and the database area are considered.

Experimental evaluation comprises TripAdvisor dataset includes 2328 restaurants with a reference of phrase from London. Extract hotel information like names and URLs total number of remarks, hotel position in the city and abrade comments for guidance to assemble textual description and obtain hotel review and reviewer data from a dataset. The surface area of the IR-tree is $10 \mathrm{~KB}$ that is recommended a fanout of 160. An LRU support thatreserves all things considered 600 pages is utilized, to expel the reserving impacts by the working framework. It drops the framework store prior to executing all queries. There by changing the proportion of query regions (default $|\mathrm{Q}|=8$ ), decide of query catchphrases (default $|\mathrm{Q}:|=8$ ), and the spatial pattern of the query lists (default 0:3\%). To measure the run of the mill runtime and I/operating system overproduced remaining tasks at hand of 80 queries for each parameter setting. 


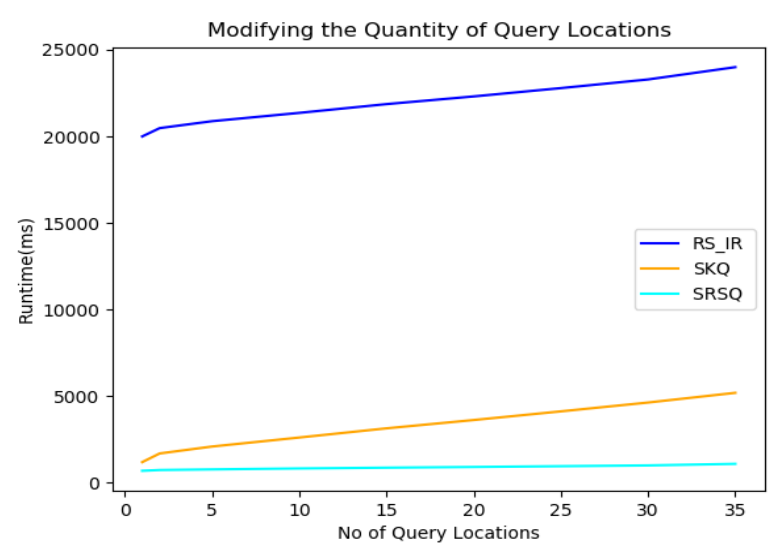

Figure 2: Modifying the Quantity of Query Locations

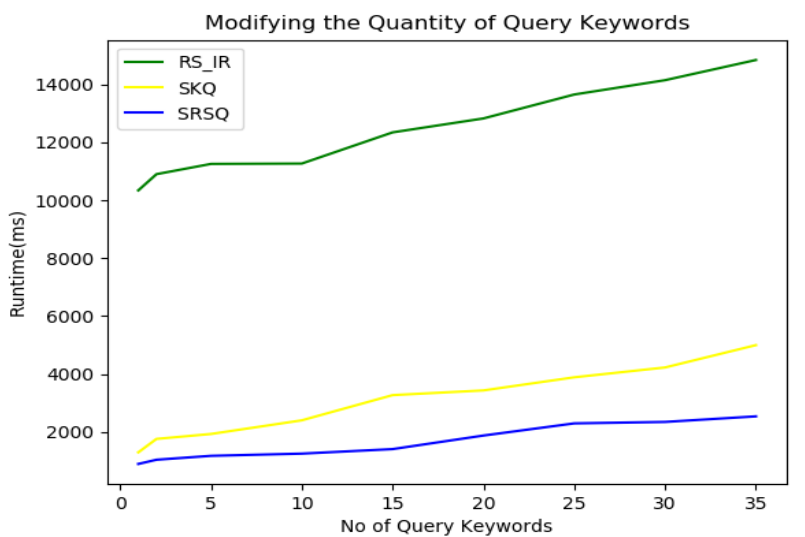

Figure 3: Modifying the Quantity of Query Keywords

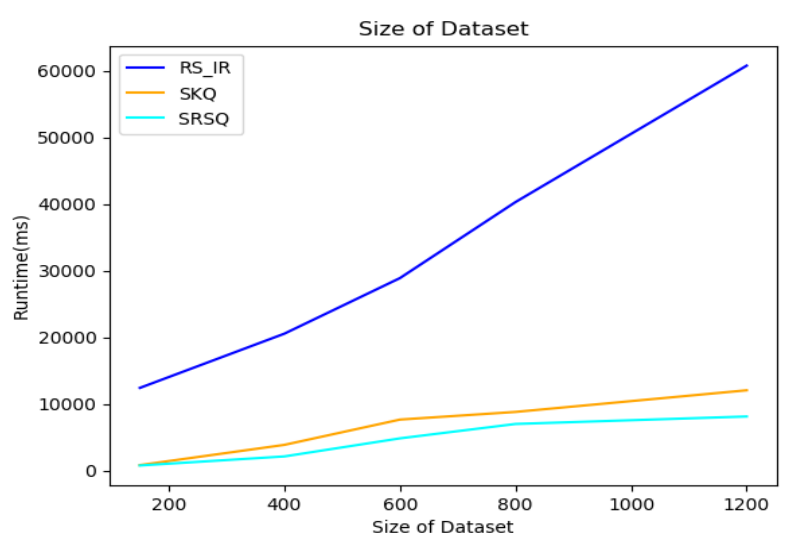

Figure 4: Size of Dataset Modifying the Quantity of Query keywords vs I/O Bar graph

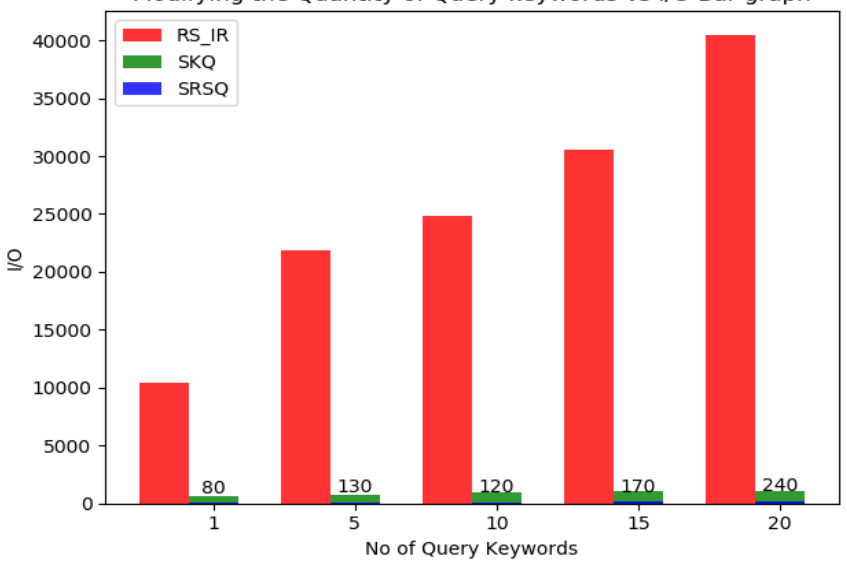

Figure 5: Modifying the Quantity of Query Keywords In the main analyze, for differ the number $|S|$ of question zones from 1 to 35 , while holding various parameters to their default regards. As Figure 2 set up, SRSK is 2 to different occasions speedier than SKQ, while SKQ beats
SKQ by a request for the measure. The I/O speeds of the three methods are identifying with this runtime. This is because of the utilization of the sky tree by SRSK and its pruning power.SKQ is quicker than RS-IR, in light of the smaller stockpiling of the posting records in the upset document and the effective procedure received for finding the required posting records. The runtime, I/O cost and the number methods increment as the quantity of query area increments since the dimensionality of the issue increments thus does the outcome size.As Figure 3 appears, the runtime of all methods by and large increments with the number of query keywords. As shown in Figures 5SKQ is faster than SR-IR. SRSK is 3 times quicker than SKQ.

\section{CONCLUSION}

In this paper a novel Spatial-Textual Skyline (STS) controlleris recommended, which notifies impressed criticalness into the spatial skyline, with the point that the recovered objects are spatially close and excellently necessary to the traffic input.RS methods based on R-tree multi-dimensional space including secondary- memory pruning tools is proposed for operating field skyline queries efficiently. After an intensive analysis of the outcomes delivered by these models, SKQ is suggestedas the best one. In particular, the skyline items conveyed by SKQ are spatially close to the mentioning zones and reasonably substance to the request watchwords. Moreover, the skyline size of SKQ is fundamentally littler contrasted with the ones of different models. Other than showing the reasonability of SKQ, algorithms for figuring the SKQ-based skyline capably are discussed. Acrucial algorithm BSTD gets an alternate leveled hybrid record,what's more, pruning benchmarks to find skyline objects determinedly. Additionally besides proposing an algorithm SRSQ that utilizes a key concept sky tree to figure the located skyline objects and also utilizes several pruning principles to diminish the predominance tests and I/O gets to brought about by SKQ. Exploratory assessment affirms the effectiveness of SRSQ over SKQ.

\section{REFERENCES}

1. G.R. Hjaltason and H. Samet, "Distance Browsing in Spatial Databases," ACM Trans. Database Systems, vol. 24, no. 2, pp. 265- 318, 1999

2. F. Korn and S. Muthukrishnan, "Influence Sets Based on Reverse Nearest Neighbor Queries," Proc. ACM SIGMOD Int'l Conf. Management of Data (SIGMOD), pp. 201-212, 2000.

3. N. Roussopoulos, S. Kelley, and F. Vincent, "Nearest Neighbor Queries," Proc. ACM SIGMOD Int'l Conf. Management of Data (SIGMOD), pp. 71-79, 1995

4. Z. Huang, H. Lu, B.C. Ooi, and A.K.H. Tung, "Continuous Skyline Queries for Moving Objects," IEEE Trans. Knowledge and Data Eng., vol. 18, no. 12, pp. 1645-1658, Dec. 2006.

5. K. Kodama, Y. Iijima, X. Guo, and Y. Ishikawa, "Skyline Queries Based on User Locations and Preferences for Making LocationBased Recommendations," Proc. Int'l Workshop Location-Based Social Networks (LBSN), pp. 9-16, 2009.

6. K.C.K. Lee, "Efficient Evaluation of Location-Dependent Skyline Queries Using Non-Dominance Scopes," Proc. Second Int'l Conf. Computing for Geospatial Research and Applications (COM.Geo), 2011

7. M.-W. Lee and S. won Hwang, "Continuous Skylining on Volatile Moving Data," Proc. DBRank: Third International Workshop on Ranking in Databases in conjunction with IEEE International Conference on Data Engineering (ICDE '09), pp. 1568-1575, 2009.

\section{Published By:}


8. L. Tian, L. Wang, P. Zou, Y. Jia, and A. Li, "Continuous Monitoring of Skyline Query over Highly Dynamic Moving Objects," Proc. Sixth ACM Int'1 Workshop Data Eng. for Wireless and Mobile Access (MobiDE), pp. 59-66, 2007

9. B. Zheng, K.C.K. Lee, and W.-C. Lee, "location-dependent Skyline Query," Proc. Ninth Int'l Conf. Mobile Data Management (MDM), pp. 148-155, 2008

10. Ihab F. Ilyas, George Beskales, and Mohamed A. Soliman. A survey of top-k query processing techniques in relational database systems. ACM Comput. Surv., 40(4):11:1-11:58, October 2008

11. A.N. Papadopoulos. Nearest Neighbor Search: A Database Perspective. Series in Computer Science. Springer, 2004.

12. Christian Bohm and Hans-Peter Kriegel. Determining the convex hull in large multidimensional databases. In Proceedings of the Third International Conference on Data Warehousing and Knowledge Discovery, DaWaK '01 pages 294-306, London, UK, UK, 2001. Springer- Verlag.

13. Franco P. Preparata and Michael Ian Shamos. Computational Geometry - An Introduction. Springer, 1985.

14. Tiakas, E., Papadopoulos, A. N., \&Manolopoulos, Y. (2015). Skyline queries An introduction. 2015 6th International Conference on Information, Intelligence, Systems and Applications (IISA).

15. Lin, X., Xu, J., \& Hu, H. (2013). Range-Based Skyline Queries in Mobile Environments. IEEE Transactions on Knowledge and Data Engineering, 25(4), 835-849.

16. Jin, W., Tung, A. K. H., Ester, M., \& Han, J. (2007). On Efficient Processing of Subspace Skyline Queries on High Dimensional Data. 19th International Conference on Scientific and Statistical Database Management (SSDBM 2007).

17. LEE, Ken C. K.; ZHENG, Baihua; CHEN, Cindy; and CHOW, Chi-Yin. Efficient Index-Based Approaches for Skyline Queries in Location-Based Applications. (2013). IEEE Transactions on Knowledge and Data Engineering. 25, (11), 2507-2520. Research Collection School Of Information Systems

18. DimitrisPapadias, Yufei Tao, Greg Fu, Bernhard Seeger "An Optimal and Progressive Algorithm for Skyline Queries"

19. Shi, J., Wu, D., \&Mamoulis, N. (2016). Textually Relevant Spatial Skylines. IEEE Transactions on Knowledge and Data Engineering, 28(1), 224-237.

20. Lim, J., Park, Y., Lee, J., Seo, D., \&Yoo, J. (2010). An efficient method for processing reverse skyline queries. 2010 Global Mobile Congress.

21. Run-Tao Liu, Xiao-Xue Chen, \& Zhen-Guo Zhao. (2014). Skyline Query algorithm based on MB-tree. 2014 International Conference on Machine Learning and Cybernetics.

22. Lee, J., Cho, H., Lee, S., \& Hwang, S. (2014). Toward Scalable Indexing for Top- $\backslash(\mathrm{k} \backslash)$ Queries. IEEE Transactions on Knowledge and Data Engineering, 26(12), 3103-3116.

23. Zhang, J., Wang, W., Jiang, X., Ku, W.-S., \& Lu, H. in 2019 An MBR-Oriented Approach for Efficient Skyline Query Processing. 2019 IEEE 35th International Conference on Data Engineering (ICDE).

24. Hsueh, Y.-L., Lin, C.-C., \& Chang, C.-C. (2017). An Efficient Indexing Method for Skyline Computations with Partially Ordered Domains. IEEE Transactions on Knowledge and Data Engineering, 29(5), 963-976.

25. Fuhry, D., Jin, R., \& Zhang, D. (2009). Efficient skyline computation in metric space. Proceedings of the 12th International Conference on Extending Database Technology Advances in Database Technology - EDBT '09.

26. Zijun Chen, ShashaGuo, and Wenyuan Liu "DIRECTION-BASED SPATIAL-TEXTUAL SKYLINE" International Journal of Innovative Computing, Information, and Control ICIC Internationa(Dc 2017 ISSN 1349-4198 Volume 13, Number 6 , December 2017

27. Li, F., Yao, B., Tang, M., \&Hadjieleftheriou, M. (2013). Spatial Approximate String Search. IEEE Transactions on Knowledge and Data Engineering, 25(6), 1394-1409.

28. Guttman, A. (1984). R-trees. Proceedings of the 1984 ACM SIGMOD International Conference on Management of Data SIGMOD '84.

29. L. Hu, W.-S. Ku, S. Bakiras, and C. Shahabi, "Spatial query integrity with voronoi neighbors," IEEE Trans. Knowl. Data Eng., vol. 25 , no. 4 , pp. 863-876, Apr. 2013

30. H. Pang, A. Jain, K. Ramamritham, and K. Tan, "Verifying completeness of relational query results in data publishing," in SIGMOD, Baltimore, MD, USA, 2005.

31. F. Li, M. Hadjieleftheriou, G. Kollios, and L. Reyzin, "Dynamic authenticated index structures for outsourced databases," in Proc.
SIGMOD, Chicago, IL, USA, 2006.

32. J. Chomicki, P. Godfrey, J. Gryz, and D. Liang, "Skyline with presorting: Theory and optimizations," in IIPWM, 2005, pp. 595604

33. D. Wu, M. L. Yiu, G. Cong, and C. S. Jensen, "Joint top-k spatial keyword query processing," IEEE TKDE, vol. 24, no. 10, pp. 1889- 1903, 2012

34. D. $\mathrm{Wu}, \mathrm{G}$. Cong, and C. S. Jensen, "A framework for efficient spatial web object retrieval," And "Spatial keyword query processing. Proceedings of the VLDB Endowment" (2013) The VLDB Journal, vol. 21, no. 6, pp. 797-822, Dec. 2012.

35. G. Cong, C. S. Jensen, and D. Wu, "Efficient retrieval of the topk most relevant spatial web objects," PVLDB, vol. 2, no. 1, pp. 337348, 2009.

36. A. Guttman. 1984. R-trees: A dynamic index structure for spatial searching. In SIGMOD Proceedings, 47-57

37. D. Papadias, Y. Tao, G. Fu, and B. Seeger. 2005. Progressive skyline computation in database systems. ACM TODS, 30(1):41-

\section{AUTHORS PROFILE}

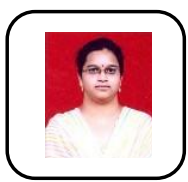

Swathi Sowmya Bavirthi, pursuing Ph.D. in JNTUH Hyderabad, Telangana State, India. She is working as Asst.Professor in IT Department, Chaitanya Bharath Institute of Technology(A), Hyderabad, Telangana State India.

Dr. Supreethi.K.P, is working as Professor in CSE Department, JNTUH College of Engineering, Hyderabad, Telangana State, India. She has 20 years of teaching experience and interested in the areas of Databases, Data Mining and Image Processing. She has 25 International Journal publications and guiding Research Scholars. 